\title{
Preoperative and Postoperative Nomograms in Predicting Early Recurrence of Hepatocellular Carcinoma Without Macrovascular Invasion After Curative Resection
}

\author{
Yanfang Zhang \\ Sichuan University \\ Liangliang Xu \\ Sichuan University \\ Mingqing Xu \\ Sichuan University \\ Hong Tang ( $\sim$ htang6198@hotmail.com ) \\ Sichuan University
}

\section{Research Article}

Keywords: Hepatocellular carcinoma (HCC), Preoperative and postoperative nomograms, macrovascular invasion, curative resection

Posted Date: September 20th, 2021

DOl: https://doi.org/10.21203/rs.3.rs-891054/v1

License: (c) (1) This work is licensed under a Creative Commons Attribution 4.0 International License.

Read Full License 


\section{Abstract}

This study aimed to establish pre- and postoperative nomograms in predicting postoperative early recurrence (ER) for hepatocellular carcinoma (HCC) without macrovascular invasion. The patients who underwent curative LR for HCC from January 2012 to December 2016 in our center were divided into training and internal prospective validation cohorts. Nomograms were constructed based on the independent risk factors derived from multivariate logistic regression analyses in training cohort. The predictive performance of nomograms was validated by internal prospective validation cohort. A total of 698 patients fulfilled with eligible criteria. Among them, 265 out of 482 patients (55.0\%) in training cohort and 120 out 216 (55.6\%) patients in validation cohort developed ER. The preoperative risk factors associated with ER were age, alpha fetoprotein (AFP), tumor diameter, tumor number; the postoperative risk factors associated with ER were age, tumor diameter, tumor number, microvasular invasion (MVI) and differentiation. The pre- and postoperative nomograms based on these factors showed good accuracy with C-indices of 0.712 and 0.850 in training cohort, and 0.754 and 0.857 in validation cohort, respectively. The calibration curves showed optimal agreement between the prediction by the nomograms and actual observation. The area under the receiver operating characteristic curves of pre- and postoperative nomograms were 0.721 and 0.848 in training cohort, and 0.754 and 0.844 in validation cohort, respectively. Present nomograms showed good performance in predicting ER for HCC without macrovascular invasion before and after surgery, which were helpful for doctors in designation of treatments and selection of patients for regularly surveillance or administration of neoadjuvant therapies.

\section{Introduction}

Hepatocellular carcinoma (HCC) is the sixth most frequency cancer and the third leading cause of cancerrelated death all over the world. ${ }^{1,2}$ Due to the prevalence of hepatitis $\mathrm{C}$ virus and alcohol intake, the incidence of HCC in western countries is increasing. ${ }^{3,4}$ Liver transplantation (LT), liver resection (LR) and radiofrequency ablation (RFA) were considered as three main curative modalities for patients with $\mathrm{HCC}$. 2,5 In clinical practice, owing to the donor shortage for LT and tumor location or diameter limitation for RFA, LR is the most common therapy for early and partial intermediate stage HCC. ${ }^{5}$ In recent years, with the remarkable improvement of surgical techniques and perioperative management, selected patients with advanced stage HCC were also could benefit from LR. ${ }^{6-9}$ Unfortunately, the dramatically high incidence of postoperative recurrence significantly decreased the survival expectancy for patients with HCC after curative LR. $2,10,11$

There are two common pattern of postoperative recurrence of HCC: early recurrence (ER) ( $<$ or $=2$ years ) deriving from occult metastasis from the initial tumor and late recurrence or de novo $\mathrm{HCC}$ ( $>2$ years after surgery) arising from underlying liver diseases. ${ }^{12-15}$ Since ER accounts for up to $70 \%$ of all postoperative recurrence and indicates poor long-term survival, more attention on ER has been drawn from researchers. ${ }^{13-15}$ On the one hand, multiple risk factors associated with ER such as microvascular invasion (MVI), high preoperative alpha fetoprotein (AFP) level, chronic active hepatitis, the absence of 
tumor capsule and large tumor size have been revealed. ${ }^{12-16}$ On the other hand, accumulate evidence showed that various postoperative adjuvant therapies such as TACE, ${ }^{17-19}$ adoptive immunotherapy, ${ }^{20}$ iodine-131-labled lipodol, ${ }^{21,22}$ interferon, ${ }^{23}$ or cancer vaccines ${ }^{24}$ could effectively delay the postoperative recurrence of $\mathrm{HCC}$, although these results need to be further verified. Therefore, indentifying patients with high risk of ER to receive appropriate adjuvant therapies might be a promising avenue in prolonging the overall survival time after curative LR.

Nomogram has been accepted by abundant investigators in predicting outcomes of various diseases. ${ }^{25-}$ ${ }^{29}$ It is constructed based on the independent risk factors of special endpoints and showed more accurate than commonly used staging systems. ${ }^{30}$ Recently, Zhang et al. ${ }^{31}$ established a nomogram which was used to predict the incidence of ER in HCC with portal vein tumor thrombus after RO LR. However, most curative LR was performed on the patients without macrovascular invasion, and the nomograms for predicting ER of this subgroup of patients is lacking. Additionally, to the best of our knowledge, no studies separately analyzed the relationship between preoperative clinical parameters and ER. And we believe a preoperative model for prediction of ER is helpful for surgeons in selecting optimal therapies for patients with high risk of postoperative ER.

In this study, independent preoperative and postoperative risk factors of ER was revealed using a large cohort of patients with HCC without macrovascular invasion. Then two nomograms were generated to predict ER before and after surgery based on these factors, respectively. The performance of these nomograms were validated by a internal prospective cohorts and receiver operating characteristic (ROC) curves.

\section{Materials And Methods}

\section{Patients}

To delete the heterogeneity on treatment of HCC from treatment concept and surgical techniques, this study only included the patients who underwent curative-intent resection for HCC from January 2012 to December 2016 in West China hospital, Sichuan University. The inclusion criteria included following items: (1) pathologically diagnosed as HCC without lymph node metastasis; (2) absence of tumor thrombus in major branches of the portal or hepatic veins; (3) receiving curative LR initially which was defined as removal of all recognizable tumor with a clear margin; (4) age not less than 18 years; (5) ChildPugh A liver function. The excluding criteria were (1) patients with other type of tumors; (2) loss of followup within 2 years; (3) poor function of other major organ (heart, lung and kidney) and (4) incomplete data. Written informed consent was obtained from each patient for data to be used for research analysis. This study was approved by ethics committee of West China Hospital, Sichuan University.

\section{Surgery}


For preparation of surgery, imaging examinations included contrast-enhanced computerized tomography (CT), and/or magnetic resonance imaging (MRI) were performed to help evaluate the characteristics of the tumor. Routine blood tests included blood cell analysis, liver/renal/coagulation function tests, hepatitis $\mathrm{B} / \mathrm{C}$ virus (HBV/HCV) screening tests, HBV deoxyribonucleic acid (HBV-DNA) measurement, serum tumor markers including alpha-fetal protein (AFP), carcinoembryonic antigen (CEA), carbohydrate antigen $19-9$ (CA $19-9)$ and CA 125 were performed. The heart and lung function were primarily evaluated by electrocardiogram and chest $\mathrm{x}$-ray, echocardiography and pulmonary function tests were added if necessary. Before surgery, a multidisciplinary team (MDT) consultation was routinely carried out to design the individual treatment for all patients. Surgical decisions were made based on tumor characteristics, reserve liver function, American Society of Anesthesiologists score ${ }^{32}$ and technological in feasibility of LR.

All eligible patients were subjected to open surgery. A right subcostal incision with midline extension was normally obtained. Intraoperative ultrasound (US) was routinely performed to seek the presence of additional nodules which were not revealed preoperatively and determine a tumor-free margin of at least $1 \mathrm{~cm}$. Anatomic resection was the first option for patients with ideal tumor location and no obvious liver cirrhosis. To decrease surgical blood loss, intermittent Pringle's maneuver was performed at a cycle of $15 / 5$ minutes of champ/unclamp time. After removal from the body, the tumor specimens were fixed with 4\% paraformaldehyde within 15 minutes and then delivered to histological department for histological examination. Finally, surgical information including surgery duration, resection type, blood loss and transfusion were recorded carefully.

\section{Follow-up}

The postoperative follow-up program has been described in our previous study. ${ }^{33}$ In brief, all patients were regularly followed up at the first postoperative month, at 3-months intervals for the subsequent 3 years, and every half year thereafter. Abdominal ultrasonography, serum AFP level, HBV-DNA load and liver function were routinely examined at each follow-up. Enhanced CT or MRI scan would be performed when suspicious lesions were found or persistent elevated AFP were measured. If necessary, bone scintigraphy or positron emission tomography was conducted to confirm bone or distant metastasis. Tumor recurrence was diagnosed based on the typical appearance of a new lesion in at least two radiological examinations, with or without elevated AFP levels. Once HCC recurrence was diagnosed, most appropriate treatments such as rehepatectomy, RFA, salvage LT, TACE, sorafenib, or best care support were recommended according to the characteristics of recurrent tumors and liver function.

Recurrence time was defined as the time interval between the operation and the first diagnosis of recurrence. In line with previous studies, we classified the patients with recurrence time no more than 2 years into ER group, otherwise, late recurrence was considered. ${ }^{12-15}$ The overall survival (OS) was defined as the time interval between the operation and death or last follow-up. The follow-up was censored in March 2019.

\section{Statistical analysis}


All statistical analyses were performed in SPSS version 24.0 (IBM SPSS Inc, Chicago, IL) and R software version 3.5.0 with the rms package (http://www.r-project.org/). Continuous variables with a normal distribution were expressed as mean (standard deviation, SD) and analyzed using Student's $t$ test or Mann-Whitney $\mathrm{U}$ test. Categorical variables were expressed as number or percentage and compared using Pearson Chi-Square or Fisher's exact test. For all included patients, the cases who underwent curative LR between January 2012 and December 2014 were allocated into the training cohort, the cases who underwent curative LR between January 2014 and December 2016 were stratified into internal prospective validation cohort. Univariate and stepwise multivariate analyses were performed using logistic regression methods to identify the independent risk factors related to ER in the training cohort. Nomograms for preoperative and postoperative prediction of ER were generated based on the results of multivariate logistic regression analyses, respectively. The predictive performance of nomograms was measured by concordance index (C-index) and calibration curves. Validation of the model performance was performed using a internal prospective validation cohort. For clinical use of present nomograms, the total pre- and postoperative risk scores of each patients were calculated using the nomograms. Receiver operating characteristic (ROC) curve analysis was performed to calculate the optimal cut off values that were determined by maximizing the Youden index (sensitivity + specificity -1$)$. The predictive ability of the optimal cut off values was assessed by the sensitivity, specificity, predictive values, and likelihood ratios. All analyses were two tailed, $p<0.05$ was considered statistically significant.

\section{Ethics approval and consent}

This study was approved by the Clinical Research Ethics Committee of West China Hospital, Chengdu, China (IRB number: FWA00009482IRBIORG0004190), and followed the guidelines outlined in the declaration of Helsinki. The study has received the informed consents from all patients.

\section{Results}

The clinical characteristics of included patients. From January 2012 to December 2016, a total of 698 patients who fulfilled with our eligible criteria were included in this study. Among them, 482 patients who underwent curative LR before January 2015 were allocated into the training cohort, the later 216 patients were stratified into internal prospective validation cohort. The detailed information of these two cohorts were listed in Table 1. Except the neutrophil-to-lymphocyte ratio (NLR) $(P=0.007)$, platelet-to-lymphocyte ratio (PLR) $(P=0.011)$ and the presence of cirrhosis $(P=0.016)$, other baseline and clinicopathologic data were comparable between the training and validation cohorts. The median follow-up for all included patients was 36 months (range, 1-78 months). The incidence of ER was observed in $265(55.0 \%)$ and $120(55.6 \%)$ patients in the training and validation cohorts, respectively. For all included patients, the 1,3 and 5-year OS rates in ER group were $66.47 \%, 23.85 \%$ and $13.18 \%$, which was dramatically lower than that in late recurrence group with the 1, 3 and 5 -year survival rate of $99.68 \%, 96.75 \%$ and $83.30 \%$, respectively $(P<0.0001)$ (Fig. 1$)$. 
Table 1

The baseline and clinical characteristics of HCC patients in the training and validation cohorts

\begin{tabular}{|c|c|c|c|}
\hline Clinical parameters & Training cohort $(n=482)$ & Validation cohort $(n=216)$ & $\mathbf{P}$ \\
\hline Gender (male/famale) & $404(83.8 \%) / 78(16.2 \%)$ & 185 (85.6\%)/31 (14.4\%) & 0.538 \\
\hline Age, mean (SD) & $49.58(12.52)$ & $51.68(10.83)$ & 0.698 \\
\hline HBsAg (positive/negative) & $417(86.5 \%) / 65(13.5 \%)$ & $187(87.0 \%) / 29(13.0 \%)$ & 0.868 \\
\hline $\begin{array}{l}\text { HBV-DNA }(\geq 103 /<103 \\
\text { copies/ml) }\end{array}$ & $261(54.2 \%) / 221(45.8 \%)$ & 107 (49.5\%)/109 (50.5\%) & 0.282 \\
\hline HBeAg (positive/negative) & 97 (20.1\%)/385 (79.9\%) & $45(21.0 \%) / 171(79.0 \%)$ & 0.323 \\
\hline $\begin{array}{l}\text { AFP }(<20 / 20-400 />400 \\
\mathrm{ng} / \mathrm{mL})\end{array}$ & $\begin{array}{l}157(32.6 \%) / 119 \\
(24.7 \%) / 206(42.7 \%)\end{array}$ & $\begin{array}{l}68(31.6 \%) / 54(25.1 \%) / 94 \\
(43.3 \%)\end{array}$ & 0.966 \\
\hline NEU $(>3.56 / \leq 3.56 \times 109 / L)$ & $228(47.4 \%) / 254(52.6 \%)$ & 99 (46.0\%)/117 (54.0\%) & 0.741 \\
\hline $\operatorname{LYM}(>1.1 / \leq 1.1 \times 109 / L)$ & $362(75.1 \%) / 120(24.9 \%)$ & $171(79.1 \%) / 45(20.9 \%)$ & 0.255 \\
\hline PLT $(>100 / \leq 100 \times 109 / L)$ & 351 (72.8\%)/131 (27.2\%) & $143(66.2 \%) / 73(33.8 \%)$ & 0.076 \\
\hline $\operatorname{NLR}(>3 / \leq 3)$ & $153(31.8 \%) / 329(68.2 \%)$ & 47 (21.9\%)/169 (78.1\%) & 0.007 \\
\hline $\operatorname{PLR}(>111 / \leq 111)$ & $180(37.3 \%) / 302(62.7 \%)$ & $59(27.4 \%) / 157$ (72.6\%) & 0.011 \\
\hline TBIL (> 28/ $\leq 28 \mathrm{umol} / \mathrm{L})$ & $15(3.1 \%) / 467$ (96.9\%) & $8(3.7 \%) / 208$ (96.3\%) & 0.689 \\
\hline $\mathrm{ALT}(>50 / \leq 50 \mathrm{IU} / \mathrm{L})$ & $161(33.4 \%) / 321(66.6 \%)$ & $76(35.2 \%) / 140(64.8 \%)$ & 0.646 \\
\hline AST $(>40 / \leq 40 \mathrm{IU} / \mathrm{L})$ & $216(44.8 \%) / 266(55.2 \%)$ & $101(46.8 \%) / 115(53.2 \%)$ & 0.633 \\
\hline $\operatorname{ALB}(>40 / \leq 40 \mathrm{~g} / \mathrm{L})$ & $308(63.9 \%) / 174(36.1 \%)$ & 131 (60.6\%)/85 (39.4\%) & 0.411 \\
\hline GGT $(>60 / \leq 60 I U / L)$ & $240(49.8 \%) / 242(50.2 \%)$ & $114(52.8 \%) / 102(47.2 \%)$ & 0.466 \\
\hline 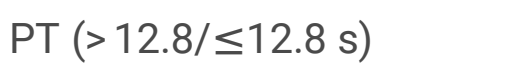 & $119(24.7 \%) / 363(75.3 \%)$ & 39 (17.9\%)/177 (82.1\%) & 0.344 \\
\hline $\operatorname{INR}(>1.15 / \leq 1.15)$ & $101(21.0 \%) / 381(79.0 \%)$ & $50(23.1 \%) / 166(76.9 \%)$ & 0.515 \\
\hline Fib $(>2 / \leq 2 \mathrm{~g} / \mathrm{L})$ & $401(83.2 \%) / 81(16.8 \%)$ & $173(79.9 \%) / 43(20.1 \%)$ & 0.338 \\
\hline $\begin{array}{l}\text { Tumor diameter }(\leq 5 / 5- \\
10 />10 \mathrm{~cm})\end{array}$ & $\begin{array}{l}219(45.4 \%) / 199(41.2 \%) / 65 \\
(13.4 \%)\end{array}$ & $\begin{array}{l}105(48.4 \%) / 89(41.2 \%) / 29 \\
(13.4 \%)\end{array}$ & 0.531 \\
\hline
\end{tabular}

Bold numbers indicate statistical significance

SD, standard deviation; HBsAg, hepatitis B surface antigen; HBV-DNA, hepatitis B virus deoxyribonucleic acid; HBeAg, hepatitis B e antigen; AFP, alpha-fetoprotein; NEU, neutrophil; LYM, lymphocyte; PLT, platelet; NLR, neutrophil-to-lymphocyte ratio; PLR, platelet-to-lymphocyte ratio; TBIL, total bilirubin; ALT, alanine transaminase; AST, aspartate aminotransferase; ALB, albumin; GGT, gamma-glutamyl transpeptidase; PT, prothrombin time; s, second; INR, international normalized ratio; Fib, fibrinogen; BCLC, Barcelona Clinic Liver Cancer staging system; MVI, microvascular invasion. 


\begin{tabular}{|c|c|c|c|}
\hline Clinical parameters & Training cohort $(n=482)$ & Validation cohort $(n=216)$ & $\mathbf{P}$ \\
\hline Tumor number (1/2/3) & $\begin{array}{l}402(83.4 \%) / 58(12.0 \%) / 22 \\
(4.6 \%)\end{array}$ & $\begin{array}{l}170(78.6 \%) / 37(17.2 \%) / 9 \\
(4.2 \%)\end{array}$ & 0.284 \\
\hline BCLC stage (A/B) & $409(84.9 \%) / 73(15.1 \%)$ & 178 (82.3\%)/38 (17.7\%) & 0.399 \\
\hline Cirrhosis (present/absent) & $296(61.4 \%) / 186(38.6 \%)$ & $153(70.8 \%) / 63(29.2 \%)$ & 0.016 \\
\hline Differentiation $(\mathrm{I}+\mathrm{II} / \mathrm{III}+\mathrm{IV})$ & $279(57.9 \%) / 203(42.1 \%)$ & $123(56.9 \%) / 93(43.1 \%)$ & 0.816 \\
\hline MVI (present/absent) & $204(42.3 \%) / 278(57.7 \%)$ & $86(39.8 \%) / 130(60.2 \%)$ & 0.534 \\
\hline $\begin{array}{l}\text { Statelite lesion } \\
\text { (present/absent) }\end{array}$ & $72(14.9 \%) / 410(85.1 \%)$ & $31(14.4 \%) / 185$ (85.6\%) & 0.840 \\
\hline $\begin{array}{l}\text { Resection (anatomic/non- } \\
\text { anatomic) }\end{array}$ & $250(51.9 \%) / 232(48.1 \%)$ & $110(50.9 \%) / 106(49.1 \%)$ & 0.818 \\
\hline \multicolumn{4}{|c|}{ Bold numbers indicate statistical significance } \\
\hline \multicolumn{4}{|c|}{$\begin{array}{l}\text { SD, standard deviation; HBsAg, hepatitis B surface antigen; HBV-DNA, hepatitis B virus } \\
\text { deoxyribonucleic acid; HBeAg, hepatitis B e antigen; AFP, alpha-fetoprotein; NEU, neutrophil; LYM, } \\
\text { lymphocyte; PLT, platelet; NLR, neutrophil-to-lymphocyte ratio; PLR, platelet-to-lymphocyte ratio; TBIL, } \\
\text { total bilirubin; ALT, alanine transaminase; AST, aspartate aminotransferase; ALB, albumin; GGT, } \\
\text { gamma-glutamyl transpeptidase; PT, prothrombin time; s, second; INR, international normalized ratio; } \\
\text { Fib, fibrinogen; BCLC, Barcelona Clinic Liver Cancer staging system; MVI, microvascular invasion. }\end{array}$} \\
\hline
\end{tabular}

The independent predictors for early recurrence. As shown in Table 2, univariate logistic analyses revealed that numerous variables including gender, age, hepatitis $B$ surface antigen (HBsAg), HBV-DNA, hepatitis B e antigen (HBeAg), AFP, neutrophil (NEU), platelet (PLT), NLR, PLR, aspartate aminotransferase (AST), gamma-glutamyl transpeptidase (GGT), tumor diameter, tumor number, Barcelona Clinic Liver Cancer (BCLC) stage, differentiation, MVI, satellite lesions and resection type were significantly associated with the ER in training cohort. Subsequent multivariate analyses further revealed that four preoperative risk factors including age (odds ratio $(\mathrm{OR})=0.894,95 \%$ confidence interval $(\mathrm{Cl})$ : $0.987-$ $0.990, P<0.001)$, AFP level (OR $=1.328,95 \% \mathrm{Cl}: 1.077-1.639, P=0.008)$, tumor diameter $(\mathrm{OR}=2.209$, $95 \% \mathrm{Cl}: 1.645-2.967, P<0.001)$ and tumor number $(\mathrm{OR}=2.556,95 \% \mathrm{Cl}: 1.474-4.434, P=0.001)$, and five postoperative risk factors including age $(\mathrm{OR}=0.981,95 \% \mathrm{Cl}: 0.975-0.987, P<0.001)$, tumor diameter (OR $=1.943,95 \% \mathrm{Cl}: 1.438-2.624, P<0.001)$, tumor number $(\mathrm{OR}=1.826,95 \% \mathrm{Cl}: 1.024-3.255, P=0.041)$, differentiation $(\mathrm{OR}=1.508,95 \% \mathrm{Cl}: 1.059-2.358, P=0.025)$ and $\mathrm{MVI}(\mathrm{OR}=2.904,95 \% \mathrm{Cl}: 1.914-4.405, P$ $<0.001)$ were significantly associated with ER of HCC patients without macrovascular invasion after curative LR (Table 3). 
Table 2

Univariate logistic analysis on clinical parameters in predicting early recurrence in the training cohort.

\section{Clinical parameters}

Gender (male/famale)

Age $(>60 / \leq 60$ years $)$

HBsAg (positive/negative)

HBV-DNA ( $\geq 103 /<103$ copies/ml)

HBeAg (positive/negative)

$\operatorname{AFP}(<20 / 20-400 />400 \mathrm{ng} / \mathrm{ml})$

NEU $(>3.56 / \leq 3.56 \times 109 / L)$

$\operatorname{LYM}(>1.1 / \leq 1.1 \times 109 / \mathrm{L})$

PLT (>100/ $\leq 100 \times 109 / L)$

$\operatorname{NLR}(>3 / \leq 3)$

PLR $(>111 / \leq 111)$

TB (>28/ $\leq 28 \mathrm{umol} / \mathrm{L})$

$\operatorname{ALT}(>50 / \leq 50 \mathrm{IU} / \mathrm{L})$

AST $(>40 / \leq 40 \mathrm{IU} / \mathrm{L})$

ALB $(>40 / \leq 40 \mathrm{~g} / \mathrm{L})$

GGT (>60/ $\leq 60 \mathrm{IU} / \mathrm{L})$

PT (>12.8/ $\leq 12.8 s)$

INR (>1.15/ $\leq 1.15)$

$\operatorname{Fib}(>2 / \leq 2 \mathrm{~g} / \mathrm{L})$

Tumor size $(\leq 5 / 5-10 />10 \mathrm{~cm})$

Tumor number $(1 / 2 / 3)$

BCLC stage (A/B)
OR ( $95 \% \mathrm{Cl})$

$1.27(1.043-1.545)$

$0.596(0.480-0.740)$

$1.262(1.075-1.482)$

1.529 (1.164-2.010)

1.771 (1.170-2.681)

1.337 (1.171-1.527)

$1.621(1.241-2.117)$

$1.221(0.993-1.502)$

$1.472(1.189-1.821)$

$2.000(1.429-2.799)$

1.687 (1.247-2.282)

$2.000(0.684-5.581)$

1.368 (1.000-1.870)

$1.602(1.218-2.108)$

1.184 (0.947-1.482)

1.697 (1.306-2.205)

1.164 (0.812-1.668)

$1.244(0.841-1.842)$

1.079 (0.873-1.335)

1.791 (1.460-2.198)

1.275 (1.108-1.466)

$3.056(1.795-5.203)$
0.017

$<0.001$

0.004

0.002

0.007

$<0.001$

$<0.001$

0.059

$<0.001$

$<0.001$

0.001

0.206

0.05

0.001

0.139

$<0.001$

0.41

0.275

0.482

$<0.001$

0.001

$<0.001$

Bold numbers indicate statistical significance

$\mathrm{OR}$, odds ratio; $\mathrm{Cl}$, confidence interval; HBsAg, hepatitis B surface antigen; HBV-DNA, hepatitis B virus deoxyribonucleic acid; HBeAg, hepatitis B e antigen; AFP, alpha-fetoprotein; NEU, neutrophil; LYM, lymphocyte; PLT, platelet; NLR, neutrophil-to-lymphocyte ratio; PLR, platelet-to-lymphocyte ratio; TBIL, total bilirubin; ALT, alanine transaminase; AST, aspartate aminotransferase; ALB, albumin; GGT, gamma-glutamyl transpeptidase; PT, prothrombin time; s, second; INR, international normalized ratio; Fib, fibrinogen; BCLC, Barcelona Clinic Liver Cancer staging system; MVI, microvascular invasion. 


\begin{tabular}{|c|c|c|}
\hline Clinical parameters & OR $(95 \% \mathrm{Cl})$ & $\mathbf{P}$ \\
\hline Cirrhosis (present/absent) & $1.193(0.949-1.499)$ & 0.131 \\
\hline Differentiation (I + II/III + IV) & $1.819(1.365-2.426)$ & $<0.001$ \\
\hline MVI (present/absent) & $2.923(2.133-4.005)$ & $<0.001$ \\
\hline Statelite lesion (present/absent) & $2.429(1.461-4.037)$ & 0.001 \\
\hline Resection (anatomic/non-anatomic) & $1.475(1.146-1.899)$ & 0.003 \\
\hline \multicolumn{3}{|c|}{ Bold numbers indicate statistical significance } \\
\hline \multicolumn{3}{|c|}{$\begin{array}{l}\text { OR, odds ratio; } \mathrm{Cl} \text {, confidence interval; HBsAg, hepatitis B surface antigen; HBV-DNA, hepatitis B virus } \\
\text { deoxyribonucleic acid; HBeAg, hepatitis B e antigen; AFP, alpha-fetoprotein; NEU, neutrophil; LYM, } \\
\text { lymphocyte; PLT, platelet; NLR, neutrophil-to-lymphocyte ratio; PLR, platelet-to-lymphocyte ratio; TBIL, } \\
\text { total bilirubin; ALT, alanine transaminase; AST, aspartate aminotransferase; ALB, albumin; GGT, } \\
\text { gamma-glutamyl transpeptidase; PT, prothrombin time; s, second; INR, international normalized ratio; } \\
\text { Fib, fibrinogen; BCLC, Barcelona Clinic Liver Cancer staging system; MVI, microvascular invasion. }\end{array}$} \\
\hline
\end{tabular}

Table 3

Multivariate logistic analysis on clinical parameters in predicting early recurrence in the training cohort.

\begin{tabular}{|lll|}
\hline Clinical parameters & OR $(95 \% \mathrm{Cl})$ & $\mathrm{P}$ \\
\hline Preoperative & & \\
\hline Age $(>60 / \leq 60$ years $)$ & $0.984(0.987-0.990)$ & $<0.001$ \\
\hline AFP $(<20 / 20-400 />400 \mathrm{ng} / \mathrm{ml})$ & $1.328(1.077-1.639)$ & 0.008 \\
\hline Tumor diameter $(\leq 5 / 5-10 />10 \mathrm{~cm})$ & $2.209(1.645-2.967)$ & $<0.001$ \\
\hline Tumor number $(1 / 2 / 3)$ & $2.556(1.474-4.434)$ & 0.001 \\
\hline Postoperative & $0.981(0.975-0.987)$ & $<0.001$ \\
\hline Age $(>60 / \leq 60$ years $)$ & $1.943(1.438-2.624)$ & $<0.001$ \\
\hline Tumor diameter $(\leq 5 / 5-10 />10 \mathrm{~cm})$ & $1.826(1.024-3.255)$ & 0.041 \\
\hline Tumor number $(1 / 2 / 3)$ & $1.580(1.059-2.358)$ & 0.025 \\
\hline Differentiation $(\mathrm{I}+\mathrm{II} / \mathrm{III}+\mathrm{IV})$ & $2.904(1.914-4.405)$ & $<0.001$ \\
\hline MVI (present/absent) & & \\
\hline OR, odds ratio; Cl, confidence interval; AFP, alpha-fetoprotein; MVI, microvascular invasion. \\
\hline
\end{tabular}

Construction of pre- and postoperative nomograms for predicting the early recurrence. Based on the results of multivariate analyses in training cohort, two nomograms for pre- or postoperatively predicting 
the ER were generated, respectively (Fig. 2). The C-indices of pre- and postoperative nomograms in training cohort were 0.712 (95\% Cl: $0.666-0.758, P<0.001)$ and $0.850(95 \% \mathrm{Cl}: 0.781-0.919, P<0.001)$, respectively. The calibration plots showed ideal agreement on the incidence of ER between the prediction by the nomograms and actual observation in follow-up (Fig. 2).

For clinical use of present nomograms, as shown in Fig. 2, the projection of each variable on the point scale meant unique score of each variable. After summing the scores of all variables, total points for each patient could be calculated. Then the projection of total points on the probability scale represented the individual probability for ER.

Validation of the prediction models. Firstly, the performance of pre- and post operative nomograms was validated by a internal prospective validation cohort. The pre- and postoperative total points for each patient in validation cohort were formulated using above two nomograms, respectively. Then the pre- or postoperative total points was treated as a new risk factor to calculate the $\mathrm{C}$-indices and produce calibration curves of ER. The results showed that the $\mathrm{C}$-indices for pre- and postoperative prediction of ER in validation cohort were 0.754 (95\% Cl: $0.690-0.818, P<0.001)$ and $0.857(95 \% \mathrm{Cl}: 0.750-0.949, P<$ $0.001)$, respectively. The calibration curves also showed ideal consistency between prediction and observation in the probability of ER (Fig. 3).

In addition, the predictive performance of present nomograms was evaluated by ROC curves (Fig. 4). In training cohort, the area under the ROC curves (AUC) of pre- and postoperative nomograms were 0.721 (95\% Cl: $0.684-0.759, P<0.001)$ and 0.848 (95\% Cl: 0.814-0.883, $P<0.001)$ respectively; in internal prospective validation cohort, the AUC of pre- and postoperative nomograms were 0.754 (95\% Cl: $0.690-$ $0.817, P<0.001)$ and 0.844 (95\% Cl: $0.790-0.897, P<0.001)$, respectively, which were comparable with the $\mathrm{C}$-indices of nomograms. These results indicated that present nomograms have good performance in predicting the ER for patients with HCC without macrovascular invasion after curative LR.

The predictive ability of nomograms. As shown in Table 4, the optimal cut-off values of the pre-and postoperative total nomogram scores were 88 (range: 4-284) and 110 (range: 5-356), respectively. For preoperative model, the sensitivity, specificity, positive predictive value, negative predictive value, positive likelihood ratio, and negative likelihood ratio in distinguishing ER were $0.611,0.716,0.704,0.587,2,151$ and 0.543 in the training cohort, and $0.730,0.677,0.733,0.674,2.260$ and 0.399 in the validation cohort. With respect for postoperative model, the sensitivity, specificity, positive predictive value, negative predictive value, positive likelihood ratio, and negative likelihood ratio in distinguishing ER were 0.706 , $0.802,0.793,0.724,3.564$ and 0.367 in the training cohort, and $0.679,0.800,0.764,0.699,3.394$ and 0.401 in the validation cohort. 
Table 4

Predictive ability of the optimal cut off values on the risk of early recurrence

\begin{tabular}{|c|c|c|c|c|}
\hline \multirow[t]{2}{*}{ variables } & \multicolumn{2}{|c|}{ Preoperative nomogram } & \multicolumn{2}{|c|}{ Postoperative nomogram } \\
\hline & $\begin{array}{l}\text { Training cohort } \\
\text { (482) }\end{array}$ & $\begin{array}{l}\text { Validation cohort } \\
\text { (216) }\end{array}$ & $\begin{array}{l}\text { Training cohort } \\
\text { (482) }\end{array}$ & $\begin{array}{l}\text { Validation cohort } \\
\text { (216) }\end{array}$ \\
\hline AUC & $\begin{array}{l}0.721(0.684- \\
0.759)\end{array}$ & $\begin{array}{l}0.754(0.690- \\
0.817)\end{array}$ & $\begin{array}{l}0.848(0.814- \\
0.883)\end{array}$ & $\begin{array}{l}0.844(0.790- \\
0.897)\end{array}$ \\
\hline Cut-off score & 88 & 88 & 110 & 110 \\
\hline Sensitivity & $\begin{array}{l}0.611(0.567- \\
0.654)\end{array}$ & $\begin{array}{l}0.642(0.578- \\
0.706)\end{array}$ & $\begin{array}{l}0.706(0.665- \\
0.747)\end{array}$ & $\begin{array}{l}0.679(0.617- \\
0.741)\end{array}$ \\
\hline Specificity & $\begin{array}{l}0.716(0.676- \\
0.756)\end{array}$ & $\begin{array}{l}0.76(0.703- \\
0.817)\end{array}$ & $\begin{array}{l}0.802(0.766- \\
0.838)\end{array}$ & $\begin{array}{l}0.800(0.746- \\
0.853)\end{array}$ \\
\hline $\begin{array}{l}\text { Positive predictive } \\
\text { value }\end{array}$ & $\begin{array}{l}0.704(0.663- \\
0.745)\end{array}$ & $\begin{array}{l}0.77(0.714- \\
0.826)\end{array}$ & $\begin{array}{l}0.793(0.756- \\
0.829)\end{array}$ & $\begin{array}{l}0.764(0.707- \\
0.821)\end{array}$ \\
\hline $\begin{array}{l}\text { Negative predictive } \\
\text { value }\end{array}$ & $\begin{array}{l}0.587(0.543- \\
0.631)\end{array}$ & $\begin{array}{l}0.629(0.565- \\
0.693)\end{array}$ & $\begin{array}{l}0.724(0.684- \\
0.764)\end{array}$ & $\begin{array}{l}0.699(0.638- \\
0.760)\end{array}$ \\
\hline $\begin{array}{l}\text { Positive likelihood } \\
\text { ratio }\end{array}$ & $\begin{array}{l}2.151(2.011- \\
2.291)\end{array}$ & $\begin{array}{l}2.675(2.393- \\
2.957)\end{array}$ & $\begin{array}{l}3.564(3.294- \\
3.834)\end{array}$ & $\begin{array}{l}3.394(3.014- \\
3.774)\end{array}$ \\
\hline $\begin{array}{l}\text { Negative likelihood } \\
\text { ratio }\end{array}$ & $\begin{array}{l}0.543(0.499- \\
0.587)\end{array}$ & $\begin{array}{l}0.471(0.404- \\
0.538)\end{array}$ & $\begin{array}{l}0.367(0.324- \\
0.411)\end{array}$ & $\begin{array}{l}0.401(0.336- \\
0.466)\end{array}$ \\
\hline
\end{tabular}

\section{Discussion}

With the development of surgical techniques and perioperative management, LR has been become more and more safe for early, most intermediate and even selected advanced stage HCC. ${ }^{34}$ And prognostic analysis showed that LR was a more effective therapy when compared with other therapies for HCC patients with advanced tumor burden. ${ }^{35,36}$ However, postoperative recurrence, especially ER, significantly shorten the survival expectancy for patients received curative LR. ${ }^{13,15,16}$ In this study, the postoperative survival time in ER group was significantly shorter than that in late recurrence group. In addition, the repeated treatments after recurrence not only seriously impacted on the living quality of patients but also heavily strengthened the medical burden.

The risk predictors for ER have been investigated by numerous studies. Imamura et al. ${ }^{12}$ found that nonanatomical resection, presence of microscopic vascular invasion and serum AFP level $\geq 32 \mathrm{ng} / \mathrm{mL}$ were significantly associated with the ER of HCC after hepatectomy. Portolani et al. ${ }^{13}$ reported that cirrhosis, chronic active hepatitis, HCV positivity, vascular infiltration and transaminases values were significantly associated with ER of HCC patients after hepatectomy. Cheng et al. ${ }^{16}$ observed that tumor diameter $>5$ $\mathrm{cm}$, the absence of a tumor capsule and the presence of microvascular invasion were correlated with ER 
of solitary HCC after curative resection. A recent study conducted by Zhang et al. ${ }^{31}$ revealed that HBV positivity, advanced type of PVTT, high HBV-DNA load, presence of satellite nodules, elevated AFP and large tumor diameter were significantly associated with ER of HCC with PVTT after R0 LR. In present study, using a large cohort of HCC patients without macrovascular invasion, four preoperative and five postoperative independent risk factors of ER were identified. The nomograms based on these factors showed good predictive ability for ER with the C-indices of 0.721 and 0.850 for pre- and postoperative models in training cohort, and 0.754 and 0.857 for pre- and postoperative models in validation cohort, respectively. Meanwhile, the calibration curves in training and validation cohorts showed ideal agreement between prediction and actual observation.

All risk factors which were incorporated in present nomograms were easily available and have been demonstrated associated with prognosis of HCC patients after curative LR. In this study, we found that the age was negatively associated with the incidence of postoperative ER. Compared with younger patients, the elderly HCC patients normally had lower AFP level, lower rate of HBsAg positivity and less tumor burden. ${ }^{37-39}$ Furthermore, the expression level of some oncogenes were significantly lower in elderly patients when compared with younger patients. ${ }^{40}$ Tumor size and numbers were commonly used as elements in various HCC staging systems. ${ }^{41-43}$ Larger tumor size and more numbers meant higher probability of intrahepatic metastasis and indicated poorer prognosis. ${ }^{44-47}$ Serum AFP level was not only a significant prognostic predictor for $\mathrm{HCC}$ patients ${ }^{48-50}$ but also associated with many metastasis characteristics of $\mathrm{HCC}$ such as $\mathrm{MVI},{ }^{51}$ incomplete tumor capsule ${ }^{52,53}$ and satellite lesions. ${ }^{54} \mathrm{MVI}$ is a discourage signal of intrahepatic vessel dissemination, ${ }^{55}$ which was repeatedly demonstrated as an independent risk factor for ER and poor OS of HCC patients who underwent curative LR. ${ }^{56-58}$ Tumors with poor pathological differentiation represents worse instinct of tumor cells, which had more powerful ability in proliferation and metastasis than tumors with well differentiation. ${ }^{59,60}$

The ROC curves showed that the optimal cut-off values for pre- and post operative nomograms were 88 and 110, respectively. Patients with a score equal or more than cut-off values meant high risk of ER. In clinical practice, present preoperative nomogram might helpful for surgeons in designation of therapy for HCC patients. The postoperative nomogram might serve as a tool to select patients for neoadjuvant therapies and more frequent surveillance.

This study had some limitations. First of all, the models of this study were constructed based on retrospective data, the performance of them need to be validated prospectively. Secondly, this study only included patients from a single center, future external validations are necessary. Thirdly, the main etiology of HCC in present study was HBV infection, the performance of present models for other etiologies-related HCC needs to be validated. Finally, other recurrence-related molecules are necessary to further improve the predictive accuracy of these nomograms.

In conclusion, Present study revealed that four preoperative and five postoperative clinical variables were significantly associated with the ER of HCC patients without macrovascular invasion after curative LR. 
Two nomograms based on these predictors showed ideal predictive performance. These prediction models were meaningful for doctors in designation of treatments before surgery and selection of patients for regular surveillance and administration of neoadjuvant therapies after surgery.

\section{Declarations}

\section{Acknowledgements}

This study was funded by the Post-Doctor Research Project, West China Hospital, Sichuan University (Nos. 2020HXBH076).

\section{Authors' contributions}

H.T, M.X conceived and designed the study; Y.Z and L.X performed all experiments; X.L responsible for the analysis and interpretation of data. Y.Z and X.L wrote the paper. All authors had read and approved the final manuscript.

\section{Competing interests}

The authors declare that they have no conflict of interest.

\section{References}

1. Torre, L. A. et al. Global cancer statistics, 2012. CA: a cancer journal for clinicians 65, 87-108, https://doi.org/10.3322/caac.21262 (2015).

2. Llovet, J. M. et al. Hepatocellular carcinoma. Nature reviews. Disease primers 2, 16018, https://doi.org/10.1038/nrdp.2016.18 (2016).

3. Bertuccio, P. et al. Global trends and predictions in hepatocellular carcinoma mortality. Journal of hepatology 67, 302-309, https://doi.org/10.1016/j.jhep.2017.03.011 (2017).

4. Siegel, R. L., Miller, K. D. \& Jemal, A. Cancer statistics, 2018. 68, 7-30, https://doi.org/10.3322/caac.21442 (2018).

5. Hartke, J., Johnson, M. \& Ghabril, M. The diagnosis and treatment of hepatocellular carcinoma. Seminars in diagnostic pathology 34, 153-159, https://doi.org/10.1053/j.semdp.2016.12.011 (2017).

6. Chok, K. S. et al. Surgical outcomes in hepatocellular carcinoma patients with portal vein tumor thrombosis. World journal of surgery 38, 490-496, https://doi.org/10.1007/s00268-013-2290-4 (2014).

7. Ye, J. Z. et al. Appropriate treatment strategies improve survival of hepatocellular carcinoma patients with portal vein tumor thrombus. World journal of gastroenterology 20, 17141-17147, https://doi.org/10.3748/wjg.v20.i45.17141 (2014).

8. Kokudo, T. et al. Survival benefit of liver resection for hepatocellular carcinoma associated with portal vein invasion. Journal of hepatology 65, 938-943, https://doi.org/10.1016/j.jhep.2016.05.044 
(2016).

9. Sakamoto, K. \& Nagano, H. Surgical treatment for advanced hepatocellular carcinoma with portal vein tumor thrombus. Hepatology research : the official journal of the Japan Society of Hepatology 47, 957-962, https://doi.org/10.1111/hepr.12923 (2017).

10. EASL-EORTC clinical practice guidelines: management of hepatocellular carcinoma. Journal of hepatology 56, 908-943, https://doi.org/10.1016/j.jhep.2011.12.001 (2012).

11. Belghiti, J., Panis, Y., Farges, O., Benhamou, J. P. \& Fekete, F. Intrahepatic recurrence after resection of hepatocellular carcinoma complicating cirrhosis. Annals of surgery 214, 114-117, https://doi.org/10.1097/00000658-199108000-00004 (1991).

12. Imamura, H. et al. Risk factors contributing to early and late phase intrahepatic recurrence of hepatocellular carcinoma after hepatectomy. Journal of hepatology 38, 200-207 (2003).

13. Portolani, N. et al. Early and late recurrence after liver resection for hepatocellular carcinoma: prognostic and therapeutic implications. Annals of surgery 243, 229-235, https://doi.org/10.1097/01.sla.0000197706.21803.a1 (2006).

14. Cucchetti, A. et al. Comparison of recurrence of hepatocellular carcinoma after resection in patients with cirrhosis to its occurrence in a surveilled cirrhotic population. Annals of surgical oncology 16, 413-422, https://doi.org/10.1245/s10434-008-0232-4 (2009).

15. Poon, R. T. Differentiating early and late recurrences after resection of HCC in cirrhotic patients: implications on surveillance, prevention, and treatment strategies. Annals of surgical oncology 16, 792-794, https://doi.org/10.1245/s10434-009-0330-y (2009).

16. Cheng, Z. et al. Risk factors and management for early and late intrahepatic recurrence of solitary hepatocellular carcinoma after curative resection. HPB : the official journal of the International Hepato Pancreato Biliary Association 17, 422-427, https://doi.org/10.1111/hpb.12367 (2015).

17. Sun, J. J. et al. Postoperative Adjuvant Transcatheter Arterial Chemoembolization After R0 Hepatectomy Improves Outcomes of Patients Who have Hepatocellular Carcinoma with Microvascular Invasion. Annals of surgical oncology 23, 1344-1351, https://doi.org/10.1245/s10434-015-5008-z (2016).

18. Liu, S. et al. Postoperative Adjuvant Trans-Arterial Chemoembolization for Patients with Hepatocellular Carcinoma and Portal Vein Tumor Thrombus. Annals of surgical oncology 25, 20982104, https://doi.org/10.1245/s10434-018-6438-1 (2018).

19. Zhang, X. P. et al. Postoperative Adjuvant Transarterial Chemoembolization Improves Outcomes of Hepatocellular Carcinoma Associated with Hepatic Vein Invasion: A Propensity Score Matching Analysis. Annals of surgical oncology 26, 1465-1473, https://doi.org/10.1245/s10434-019-07223-z (2019).

20. Takayama, T. et al. Adoptive immunotherapy to lower postsurgical recurrence rates of hepatocellular carcinoma: a randomised trial. Lancet (London, England) 356, 802-807, https://doi.org/10.1016/s0140-6736(00)02654-4 (2000). 
21. Lau, W. Y., Lai, E. C., Leung, T. W. \& Yu, S. C. Adjuvant intra-arterial iodine-131-labeled lipiodol for resectable hepatocellular carcinoma: a prospective randomized trial-update on 5-year and 10-year survival. Annals of surgery 247, 43-48, https://doi.org/10.1097/SLA.0b013e3181571047 (2008).

22. Boucher, E. et al. Adjuvant intra-arterial injection of iodine-131-labeled lipiodol after resection of hepatocellular carcinoma. Hepatology (Baltimore, Md.) 38, 1237-1241, https://doi.org/10.1053/jhep.2003.50473 (2003).

23. Sun, H. C. et al. Postoperative interferon alpha treatment postponed recurrence and improved overall survival in patients after curative resection of HBV-related hepatocellular carcinoma: a randomized clinical trial. Journal of cancer research and clinical oncology 132, 458-465, https://doi.org/10.1007/s00432-006-0091-y (2006).

24. Peng, B. G. et al. Tumor vaccine against recurrence of hepatocellular carcinoma. World journal of gastroenterology 11, 700-704, https://doi.org/10.3748/wjg.v11.i5.700 (2005).

25. He, Y. et al. Development and Validation of a Novel Diagnostic Nomogram to Differentiate Between Intestinal Tuberculosis and Crohn's Disease: A 6-year Prospective Multicenter Study. The American journal of gastroenterology 114, 490-499, https://doi.org/10.14309/ajg.0000000000000064 (2019).

26. Wang, Y. et al. Prognostic nomogram for intrahepatic cholangiocarcinoma after partial hepatectomy. Journal of clinical oncology : official journal of the American Society of Clinical Oncology 31, 11881195, https://doi.org/10.1200/jco.2012.41.5984 (2013).

27. Gross, J. P. et al. Development and validation of a nomogram to predict lymphedema following axillary surgery and radiotherapy in women with breast cancer from the NCIC CTG MA.20 randomized trial. International journal of radiation oncology, biology, physics, https://doi.org/10.1016/j.jijobp.2019.05.002 (2019).

28. Chon, Y. E. et al. Development of a New Nomogram Including Neutrophil-to-Lymphocyte Ratio to Predict Survival in Patients with Hepatocellular Carcinoma Undergoing Transarterial Chemoembolization. 11, https://doi.org/10.3390/cancers11040509 (2019).

29. Nassiri, F. et al. DNA methylation profiling to predict recurrence risk in meningioma: development and validation of a nomogram to optimize clinical management. Neuro-oncology, https://doi.org/10.1093/neuonc/noz061 (2019).

30. Balachandran, V. P., Gonen, M., Smith, J. J. \& DeMatteo, R. P. Nomograms in oncology: more than meets the eye. The Lancet. Oncology 16, e173-180, https://doi.org/10.1016/s1470-2045(14)71116-7 (2015).

31. Zhang, X. P. et al. A nomogram to predict early postoperative recurrence of hepatocellular carcinoma with portal vein tumour thrombus after R0 liver resection: A large-scale, multicenter study. European journal of surgical oncology : the journal of the European Society of Surgical Oncology and the British Association of Surgical Oncology, https://doi.org/10.1016/j.ejso.2019.03.043 (2019).

32. Owens, W. D., Felts, J. A. \& Spitznagel, E. L., Jr. ASA physical status classifications: a study of consistency of ratings. Anesthesiology 49, 239-243 (1978). 
33. Xu, L. et al. Novel Prognostic Nomograms for Hepatocellular Carcinoma Patients with Microvascular Invasion: Experience from a Single Center. Gut and liver, https://doi.org/10.5009/gnl18489 (2019).

34. Vitale, A. et al. Survival benefit of liver resection for patients with hepatocellular carcinoma across different Barcelona Clinic Liver Cancer stages: a multicentre study. Journal of hepatology 62, 617624, https://doi.org/10.1016/j.jhep.2014.10.037 (2015).

35. Zhong, J. H. et al. Hepatic resection associated with good survival for selected patients with intermediate and advanced-stage hepatocellular carcinoma. Annals of surgery 260, 329-340, https://doi.org/10.1097/sla.0000000000000236 (2014).

36. Hsu, C. Y. et al. Surgical Resection is Better than Transarterial Chemoembolization for Patients with Hepatocellular Carcinoma Beyond the Milan Criteria: A Prognostic Nomogram Study. Annals of surgical oncology 23, 994-1002, https://doi.org/10.1245/s10434-015-4929-x (2016).

37. Faber, W. et al. Significant impact of patient age on outcome after liver resection for HCC in cirrhosis. European journal of surgical oncology : the journal of the European Society of Surgical Oncology and the British Association of Surgical Oncology 40, 208-213, https://doi.org/10.1016/j.ejso.2013.10.018 (2014).

38. Huang, J. et al. Long-term outcomes and prognostic factors of elderly patients with hepatocellular carcinoma undergoing hepatectomy. Journal of gastrointestinal surgery : official journal of the Society for Surgery of the Alimentary Tract 13, 1627-1635, https://doi.org/10.1007/s11605-009-09334 (2009).

39. Motoyama, $\mathrm{H}$. et al. Impact of advanced age on the short- and long-term outcomes in patients undergoing hepatectomy for hepatocellular carcinoma: a single-center analysis over a 20-year period. American journal of surgery 209, 733-741, https://doi.org/10.1016/j.amjsurg.2014.05.026 (2015).

40. Yan, H. et al. Characterization of the genotype and integration patterns of hepatitis B virus in earlyand late-onset hepatocellular carcinoma. Hepatology (Baltimore, Md.) 61, 1821-1831, https://doi.org/10.1002/hep.27722 (2015).

41. Llovet, J. M., Bru, C. \& Bruix, J. Prognosis of hepatocellular carcinoma: the BCLC staging classification. Seminars in liver disease 19, 329-338, https://doi.org/10.1055/s-2007-1007122 (1999).

42. Minagawa, M., Ikai, I., Matsuyama, Y., Yamaoka, Y. \& Makuuchi, M. Staging of hepatocellular carcinoma: assessment of the Japanese TNM and AJCC/UICC TNM systems in a cohort of 13,772 patients in Japan. Annals of surgery 245, 909-922, https://doi.org/10.1097/01.sla.0000254368.65878.da (2007).

43. Yau, T. et al. Development of Hong Kong Liver Cancer staging system with treatment stratification for patients with hepatocellular carcinoma. Gastroenterology 146, 1691-1700.e1693, https://doi.org/10.1053/j.gastro.2014.02.032 (2014).

44. Chiappa, A. et al. Factors affecting survival and long-term outcome in the cirrhotic patient undergoing hepatic resection for hepatocellular carcinoma. European journal of surgical oncology : 
the journal of the European Society of Surgical Oncology and the British Association of Surgical Oncology 26, 387-392, https://doi.org/10.1053/ejso.1999.0904 (2000).

45. Fu, Y. P. et al. Prognostic Nomograms Stratify Survival of Patients with Hepatocellular Carcinoma Without Portal Vein Tumor Thrombosis After Curative Resection. The oncologist 22, 561-569, https://doi.org/10.1634/theoncologist.2016-0231 (2017).

46. Izumi, R. et al. Prognostic factors of hepatocellular carcinoma in patients undergoing hepatic resection. Gastroenterology 106, 720-727 (1994).

47. Nathan, H., Schulick, R. D., Choti, M. A. \& Pawlik, T. M. Predictors of survival after resection of early hepatocellular carcinoma. Annals of surgery 249, 799-805, https://doi.org/10.1097/SLA.0b013e3181a38eb5 (2009).

48. Ma, W. J., Wang, H. Y. \& Teng, L. S. Correlation analysis of preoperative serum alpha-fetoprotein (AFP) level and prognosis of hepatocellular carcinoma (HCC) after hepatectomy. World journal of surgical oncology 11, 212, https://doi.org/10.1186/1477-7819-11-212 (2013).

49. Allard, M. A. et al. The postresection alpha-fetoprotein in cirrhotic patients with hepatocellular carcinoma. An independent predictor of outcome. Journal of gastrointestinal surgery : official journal of the Society for Surgery of the Alimentary Tract 18, 701-708, https://doi.org/10.1007/s11605-0132433-9 (2014).

50. Zhang, X. F. et al. Changes of serum alpha-fetoprotein and alpha-fetoprotein-L3 after hepatectomy for hepatocellular carcinoma: prognostic significance. Hepatobiliary \& pancreatic diseases international : HBPD INT 11, 618-623 (2012).

51. Lei, Z. et al. Nomogram for Preoperative Estimation of Microvascular Invasion Risk in Hepatitis B Virus-Related Hepatocellular Carcinoma Within the Milan Criteria. JAMA surgery 151, 356-363, https://doi.org/10.1001/jamasurg.2015.4257 (2016).

52. Xu, J. et al. Distinctions between clinicopathological factors and prognosis of alpha-fetoprotein negative and positive hepatocelluar carcinoma patients. Asian Pacific journal of cancer prevention : APJCP 13, 559-562, https://doi.org/10.7314/apjcp.2012.13.2.559 (2012).

53. Tada, T. et al. Relationship between Lens culinaris agglutinin-reactive alpha-fetoprotein and pathologic features of hepatocellular carcinoma. Liver international : official journal of the International Association for the Study of the Liver 25, 848-853, https://doi.org/10.1111/j.14783231.2005.01111.x (2005).

54. Maeda, T. et al. Small hepatocellular carcinoma with minute satellite nodules. Hepatogastroenterology 47, 1063-1066 (2000).

55. Rodriguez-Peralvarez, M. et al. A systematic review of microvascular invasion in hepatocellular carcinoma: diagnostic and prognostic variability. Annals of surgical oncology 20, 325-339, https://doi.org/10.1245/s10434-012-2513-1 (2013).

56. Fan, S. T. et al. Outcome after partial hepatectomy for hepatocellular cancer within the Milan criteria. The British journal of surgery 98, 1292-1300, https://doi.org/10.1002/bjs.7583 (2011). 
57. Kaibori, M., Ishizaki, M., Matsui, K. \& Kwon, A. H. Predictors of microvascular invasion before hepatectomy for hepatocellular carcinoma. Journal of surgical oncology 102, 462-468, https://doi.org/10.1002/jso.21631 (2010).

58. Wang, C. C. et al. Perioperative factors affecting long-term outcomes of 473 consecutive patients undergoing hepatectomy for hepatocellular carcinoma. Annals of surgical oncology 16, 1832-1842, https://doi.org/10.1245/s10434-009-0448-y (2009).

59. Han, D. H. et al. Prognostic significance of the worst grade in hepatocellular carcinoma with heterogeneous histologic grades of differentiation. Journal of gastroenterology and hepatology 28, 1384-1390, https://doi.org/10.1111/jgh.12200 (2013).

60. Sasaki, K. et al. In hepatocellular carcinomas, any proportion of poorly differentiated components is associated with poor prognosis after hepatectomy. World journal of surgery 38, 1147-1153, https://doi.org/10.1007/s00268-013-2374-1 (2014).

\section{Figures}


Figure 1

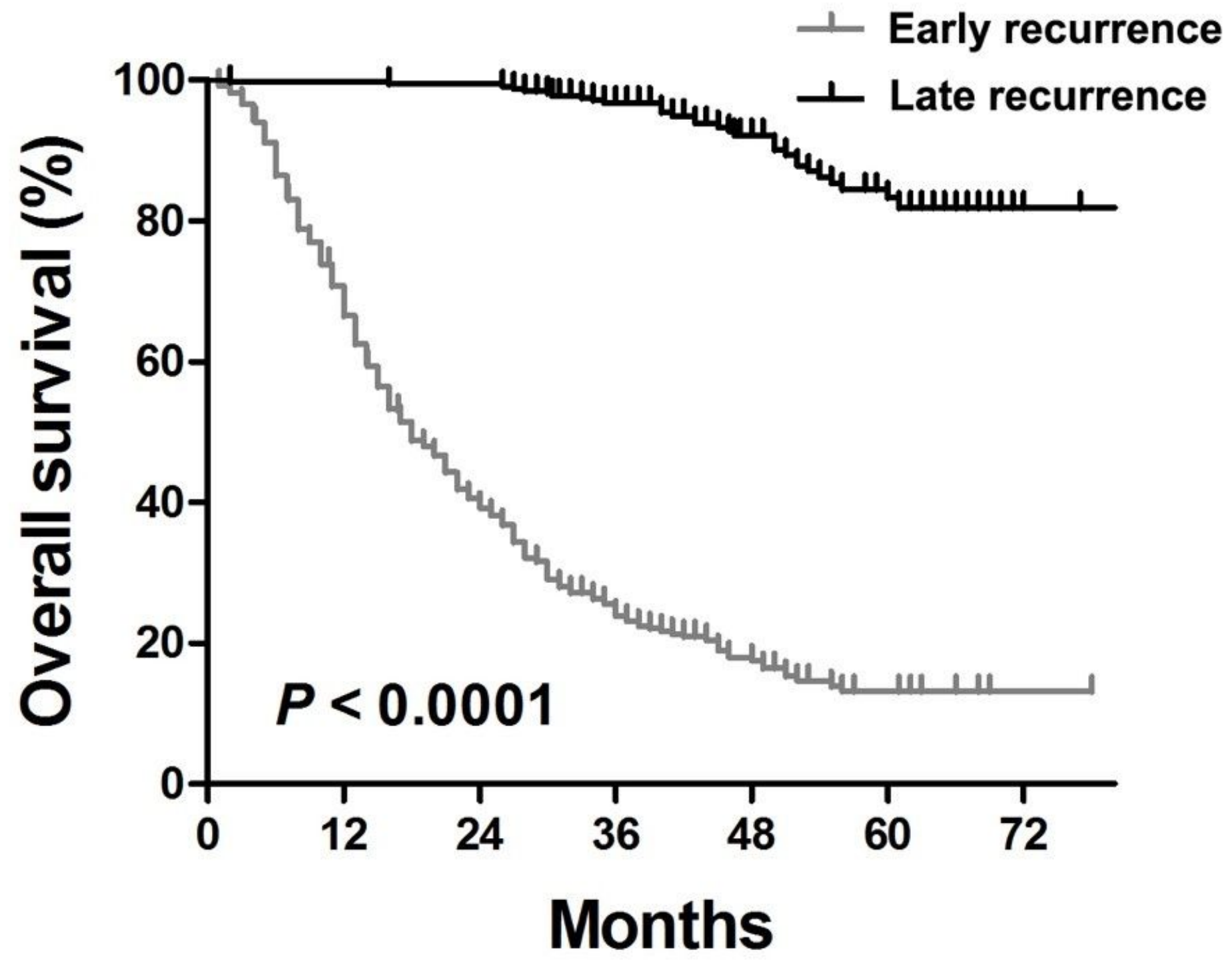

Figure 1

The overall survival rate of patients with hepatocellular carcinoma in Early (ER) and late recurrence groups. 


\section{Figure 2}

A

Point

Age (years)

$\operatorname{AFP}(n g / m L)$

Tumor diameter $(\mathrm{cm})$

Tumor number

Total points

Probability of Early Recurrence

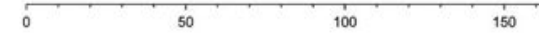

0.2

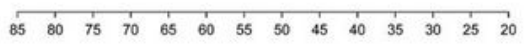

20-400
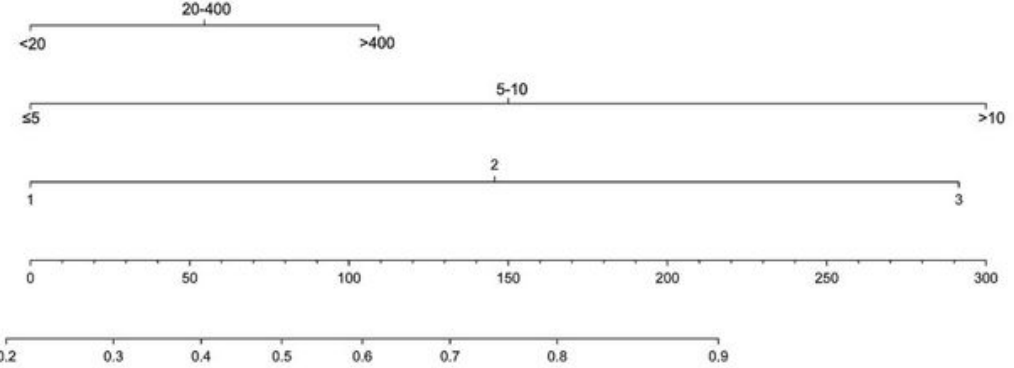

B

Point

Age (years)

Tumor diameter $(\mathrm{cm})$

Tumor number

MVI

Differentiation

Total points

Probability of Early Recurrence

C

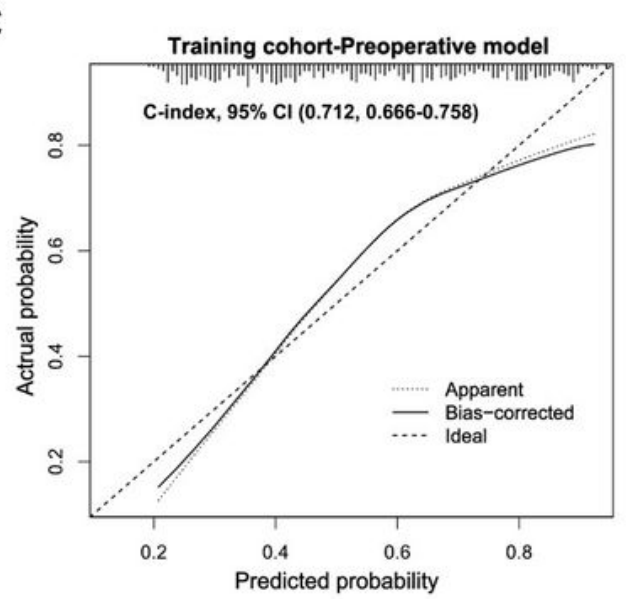

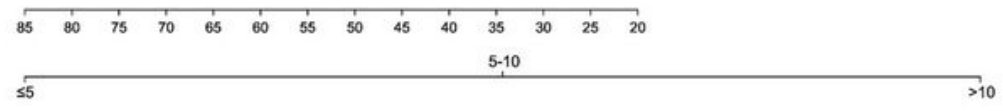

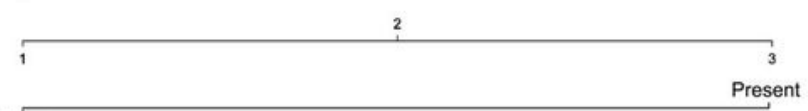

Absent

Absent

Present

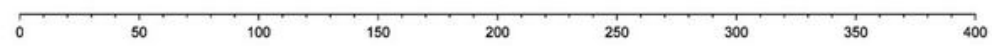

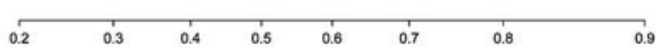

D

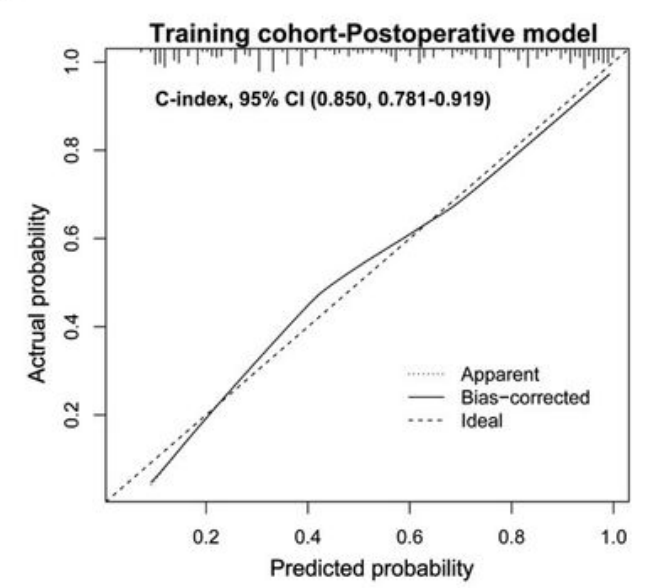

\section{Figure 2}

Pre- and postoperative nomograms and calibration curves for predicting early recurrence in training cohort. (a) The preoperative independent risk factors and nomogram for early recurrence. (b) The postoperative independent risk factors and nomogram for early recurrence. (c) The calibration curve of preoperative nomogram in training cohort. (d) The calibration curve of postoperative nomogram in raining cohort. 


\section{Figure 3}

A

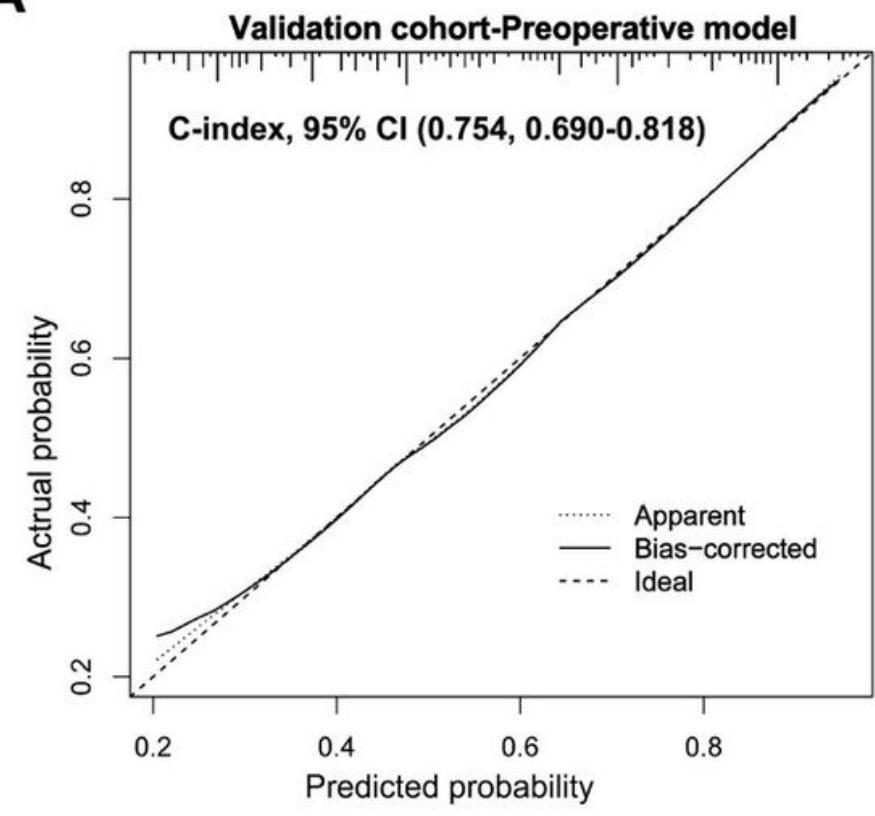

B

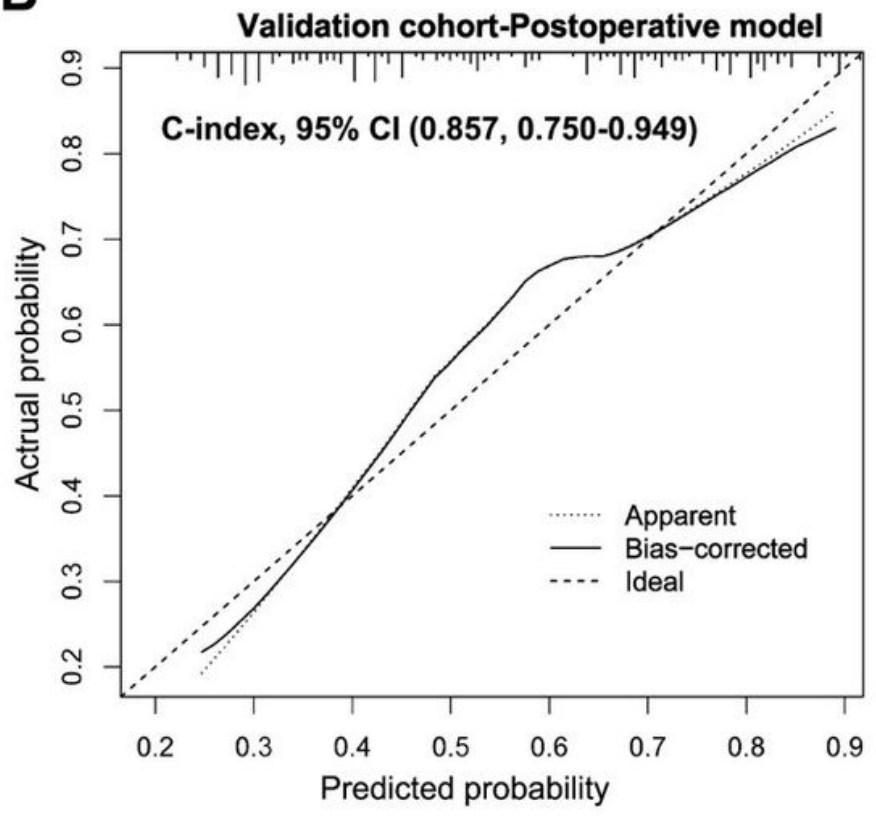

Figure 3

The calibration curves of pre-and postoperative nomograms in internal prospective validation cohort. 


\section{Figure 4}

A

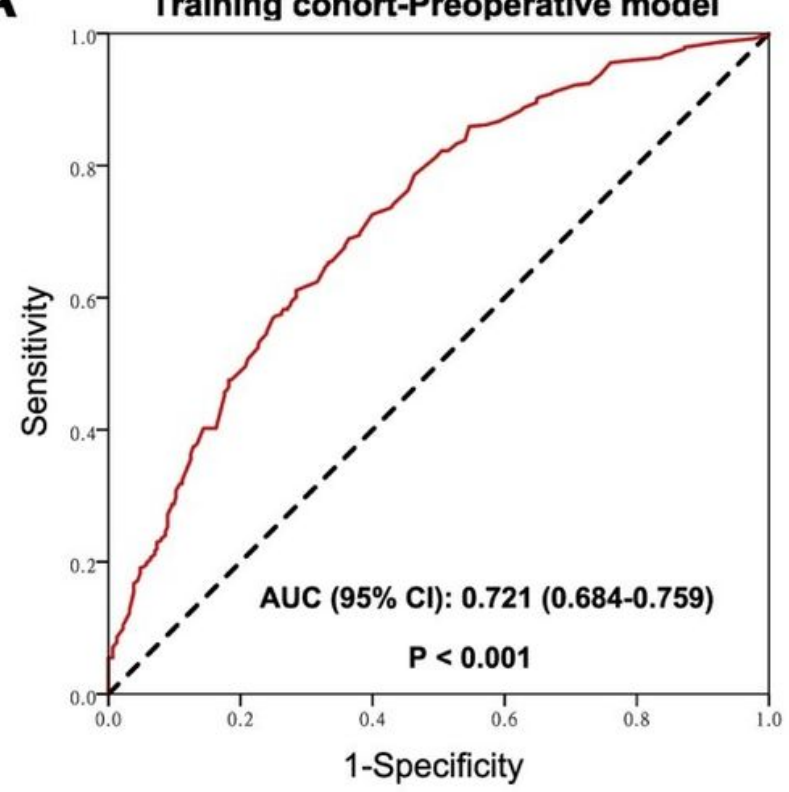

C

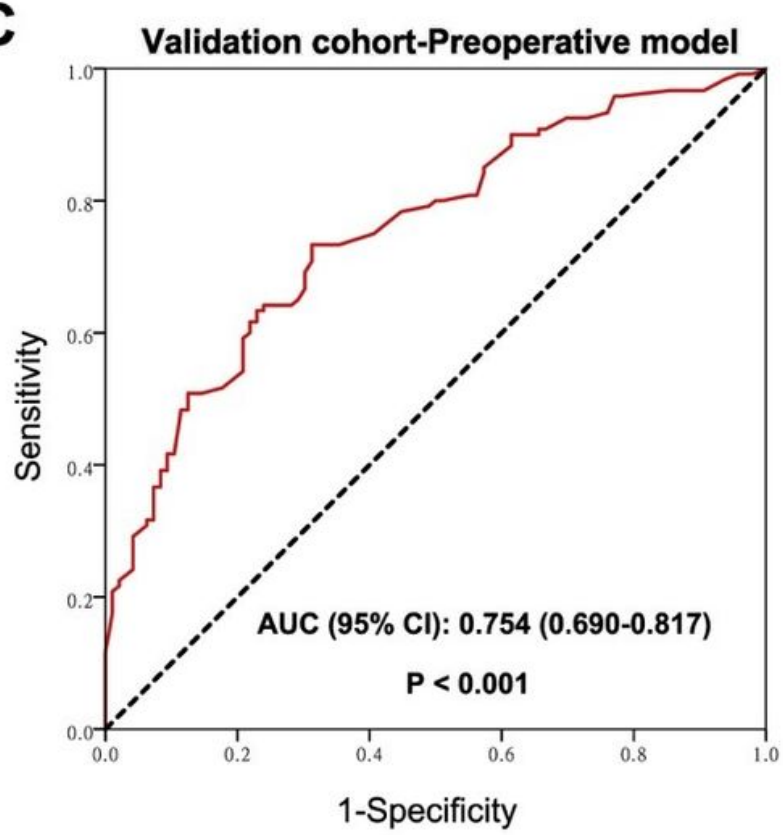

B

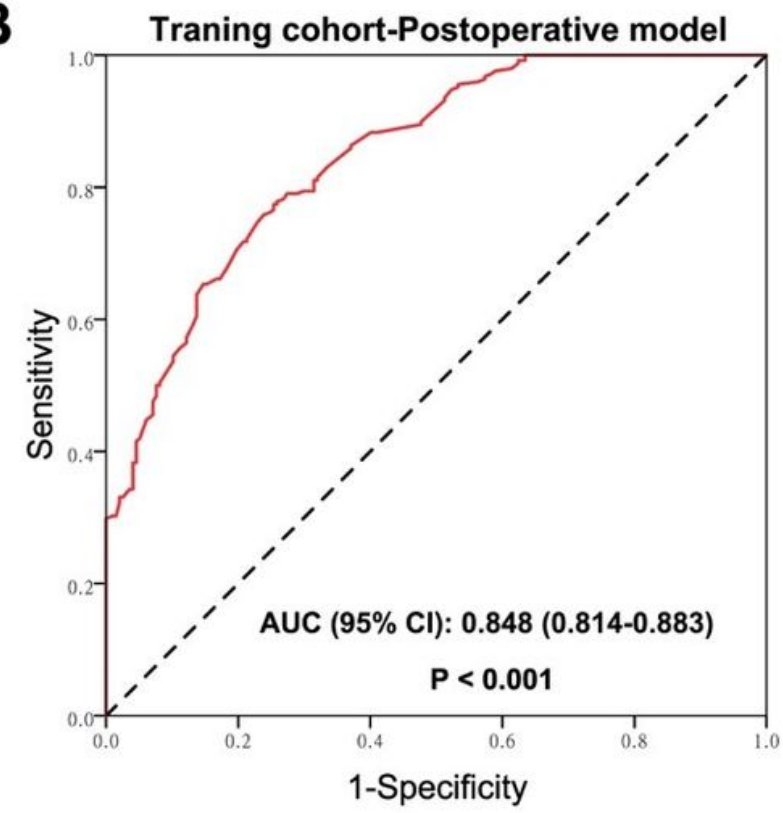

D

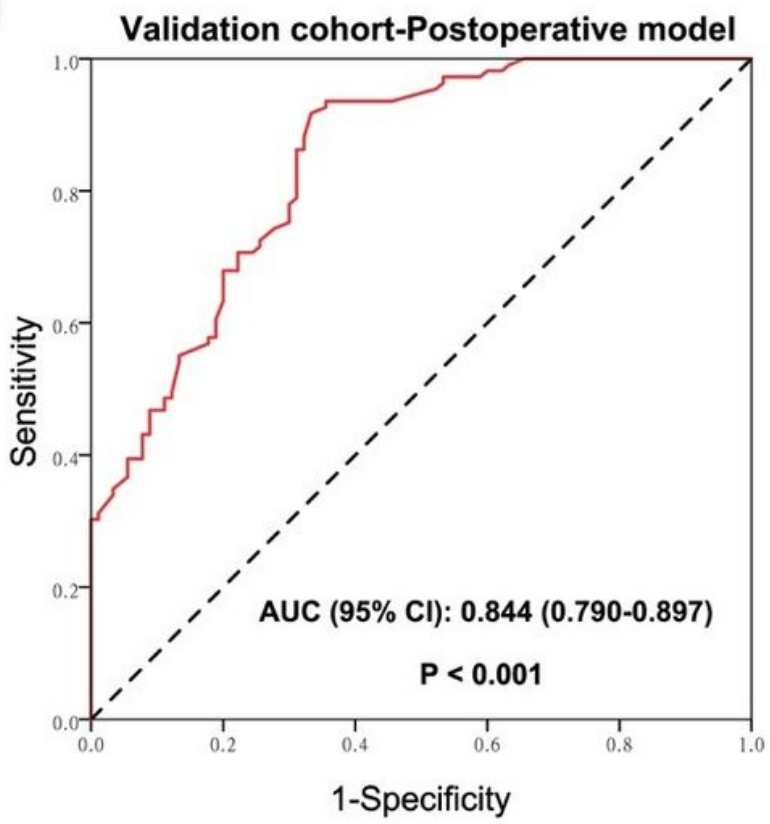

\section{Figure 4}

The receiver operating characteristic curves of pre- and postoperative nomograms in training and validation cohorts. 